Electronic Supplementary Information (ESI)

\title{
Water Solubility and Surface Activity of Alkoxyethyl $\beta$-D-Maltosides
}

Fang Fu, Yulin Fan, Langqiu Chen, ${ }^{*}$ Jing Zhang, and Jiping Li Key Laboratory of Environmentally Friendly Chemistry and Application of Ministry of Education, College of Chemistry, Xiangtan University, Xiangtan, Hunan, 411105, People's Republic of China

\section{Content}

1. Synthesis routine of alkoxyethyl $\beta$-D-maltosides.................................S2

2. General methods..............................................................S2

3. Synthesis of alkoxyethyl $\beta$-D-maltosides............................................S2

4. Data of hexoxyethyl $2,3,6,2^{\prime}, 3^{\prime}, 4^{\prime}$ '.6'-hepta- $O$-acetyl- $\beta$ - $D$-maltoside (3a)........ S3

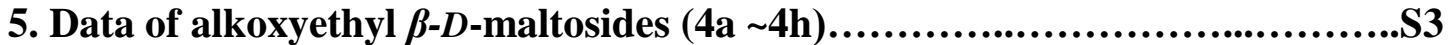

*E-mail: chengood2003@263.net 


\section{Synthesis routine of alkoxyethyl $\beta$ - $D$-maltosides}

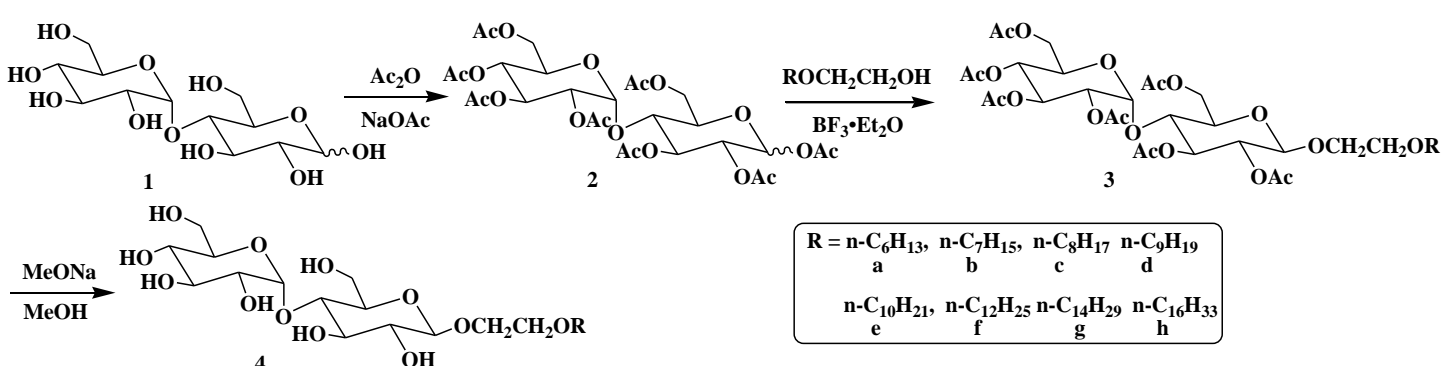

Figure S1. Synthesis of alkoxyethyl $\beta$ - $D$-maltosides

\section{General methods}

The purity of all raw materials and reagents was chemical pure or analytical pure. ${ }^{1} \mathrm{H}$ NMR and HH COSY were determined with Bruker AVANCE III HD $400 \mathrm{MHz}$ NMR Spectrometer with DMSO- $d_{6}$, DMSO- $d_{6} / \mathrm{D}_{2} \mathrm{O}, \mathrm{D}_{2} \mathrm{O}$, or chloroform- $d\left(\mathrm{CDCl}_{3}\right)$ as the solvents and tetramethylsilane (TMS) as the internal standard. Optical rotations were obtained on a Perkin-Elmer model 341-MC automatic polarimeter for solutions in a $1-\mathrm{dm}$, jacketed cell. High resolution mass spectrum (HRMS) was determined with Thermo Scientific LTQ Orbitrap XL with ESI as ion resource. All reaction processes were monitored with thin-layer chromatography (TLC). The TLC was performed on silica gel $\mathrm{HF}_{254}$ with detection by charring with a mixture of $30 \%(\mathrm{v} / \mathrm{v})$ sulfuric acid and methanol.

\section{Synthesis of alkoxyethyl $\beta$ - $D$-maltosides}

With $D$-maltose as raw material, acetylation was carried in the presence of acetic anhydride and anhydrous sodium acetate to provide octaacetylmaltose (2) in $83.1 \%$ yield. Octaacetylmaltose 2 (10.00 g, $14.74 \mathrm{mmol})$ was dissolved in anhydrous dichloromethane $\left(\mathrm{CH}_{2} \mathrm{Cl}_{2}, 100 \mathrm{~mL}\right)$, followed by the addition of ethylene glycol monohexylether $(3.23 \mathrm{~g}, 22.11 \mathrm{mmol})$, and then $\mathrm{BF}_{3} \cdot \mathrm{Et}_{2} \mathrm{O}(7.44 \mathrm{~mL}, 58.96 \mathrm{mmol})$ was dropwise added into the mixture while stirring at $0{ }^{\circ} \mathrm{C}$ for $30 \mathrm{~min}$. The mixture was placed at room temperature under stirring for $17 \mathrm{~h}$, and the reaction progress was monitored by TLC method (petroleum ether : ethyl acetate $=1: 1$ ). After the reaction was completed, the mixture was quenched by saturated aq. $\mathrm{NaHCO}_{3}$ solution. After extracted with $\mathrm{CH}_{2} \mathrm{Cl}_{2}$, the solution was dried with anhydrous $\mathrm{Na}_{2} \mathrm{SO}_{4}$ for $2 \mathrm{~h}$. The filtrate was evaporated to dryness under reduced pressure. The residue was purified 
by a flash column chromatography (petroleum ether : ethyl acetate $=3: 1$ ) to give a brown syrup hexoxyethyl 2,3,6,2',3',4'6'-hepta- $O$-acetyl- $\beta$ - $D$-maltoside (3a) $(5.82 \mathrm{~g}$, yield $51.6 \%$ ).

Compound 3a (5.00 g, $6.54 \mathrm{mmol})$ was dissolved in methanol $(80 \mathrm{~mL})$, and 15\% sodium methoxide/methanol solution was added dropwise to adjust the $\mathrm{pH}$ value 9.5 10.5. The mixture was stirred for $2 \mathrm{~h}$, and the reaction was completed by TLC (ethyl acetate: methanol $=3: 1$ ) method. The mixture was neutralized to adjust the $\mathrm{pH}$ value 7 by using acetic acid. The mixture was concentrated under the reduced pressure. The residue was purified by a silica gel chromatography (ethyl acetate: methanol $=8: 1$ ) to achieve hexoxyethyl $\beta$ - $D$-maltoside (4a) (2.52 g, yield 81.9\%).

Other alkoxyethyl $\beta$ - $D$-maltosides (4b, 4c, 4d, 4e, 4f, 4g, 4h) (n = 7, 8, 9, 10, 12, $14,16)$ were also prepared by using the similar method.

\section{Data of hexoxyethyl $2,3,6,2^{\prime}, 3^{\prime}, 4^{\prime}$ '.6'-hepta- $O$-acetyl- $\beta$ - $D$-maltoside (3a)}

$[\alpha]_{\mathrm{D}}^{20}+45.4^{\mathrm{o}}\left(\mathrm{c} 1.0, \mathrm{CH}_{3} \mathrm{OH}\right) .{ }^{1} \mathrm{H} \mathrm{NMR}\left(\mathrm{CDCl}_{3}\right): \delta 5.41\left(\mathrm{~d}, 1 \mathrm{H}, J_{1,2}=4.0 \mathrm{~Hz}\right.$, H-1'), $5.36\left(\mathrm{dd}, 1 \mathrm{H}, J_{2,3}=J_{3,4}=10.0 \mathrm{~Hz}, \mathrm{H}-3\right.$ ') $5.25\left(\mathrm{dd}, 1 \mathrm{H}, J_{2,3}=J_{3,4}=9.1 \mathrm{~Hz}\right.$, H-3), 5.06 (dd, 1H, J,5 =9.9 Hz, H-4'), 4.86 (dd, 1H, H-2'), 4.83 (dd, 1H, H-2), 4.63 $\left(\mathrm{d}, 1 \mathrm{H}, J_{1,2}=7.9 \mathrm{~Hz}, \mathrm{H}-1\right), 4.48\left(\mathrm{dd}, 1 \mathrm{H}, J_{5,6 \mathrm{a}}=2.7 \mathrm{~Hz}, J_{6 \mathrm{a}, 6 \mathrm{~b}}=12.0 \mathrm{~Hz}, \mathrm{H}-6 \mathrm{a}\right), 4.26$

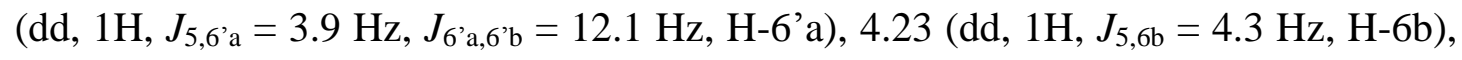
$4.04\left(\mathrm{dd}, 1 \mathrm{H}, J_{5,6^{\prime} \mathrm{b}}=2.1 \mathrm{~Hz}, \mathrm{H}-6\right.$ 'b), 4.01 (dd, 1H, $\left.J_{4,5}=9.1 \mathrm{~Hz}, \mathrm{H}-4\right), 3.94 \sim 3.98$ (m, $\left.1 \mathrm{H}, \quad \mathrm{H}-5^{\prime}\right), 3.89 \sim 3.94\left(\mathrm{~m}, 1 \mathrm{H}, \mathrm{OCH}_{2} \mathrm{CH}_{2} \mathrm{OC}_{6} \mathrm{H}_{13}\right), 3.69 \sim 3.74(\mathrm{~m}, 1 \mathrm{H}$, $\left.\mathrm{OCH}_{2} \mathrm{CH}_{2} \mathrm{OC}_{6} \mathrm{H}_{13}\right), 3.66 \sim 3.70(\mathrm{~m}, 1 \mathrm{H}, \mathrm{H}-5), 3.53 \sim 3.59\left(\mathrm{~m}, 2 \mathrm{H}, \mathrm{OCH}_{2} \mathrm{CH}_{2} \mathrm{OC}_{6} \mathrm{H}_{13}\right)$, $3.42\left(\mathrm{t}, 2 \mathrm{H}, J=6.7 \mathrm{~Hz}, \mathrm{OCH}_{2}\left(\mathrm{CH}_{2}\right)_{4} \mathrm{CH}_{3}\right), 2.15$ (s, 3H, Ac), 2.11 (s, 3H, Ac), 2.05 (s, 3H, Ac), 2.03 (s, 3H, Ac), 2.02 (s, 3H, Ac), 2.00 (s, 6H, 2Ac), $1.52 \sim 1.58(\mathrm{~m}, 2 \mathrm{H}$, $\left.\mathrm{OCH}_{2} \mathrm{CH}_{2}\left(\mathrm{CH}_{2}\right)_{3} \mathrm{CH}_{3}\right), 1.25 \sim 1.34\left(\mathrm{~m}, 6 \mathrm{H}, \mathrm{O}\left(\mathrm{CH}_{2}\right)_{2}\left(\mathrm{CH}_{2}\right)_{3} \mathrm{CH}_{3}\right), 0.89(\mathrm{t}, 3 \mathrm{H}, J=6.8$ $\mathrm{Hz}, \mathrm{CH}_{3}$ ). HRMS (ESI) m/z: calcd for $\mathrm{C}_{34} \mathrm{H}_{52} \mathrm{O}_{19} \mathrm{Na}^{+}(\mathrm{M}+\mathrm{Na})^{+} 787.29950$, found 787.29944.

\section{Data of alkoxyethyl $\beta$-D-maltosides $(4 a \sim 4 h)$}

\subsection{Hexoxyethyl $\beta$ - $D$-maltoside}

$[\alpha]_{\mathrm{D}}^{20}+57.4^{\circ}\left(\mathrm{c} 1.0, \mathrm{CH}_{3} \mathrm{OH}\right) .{ }^{1} \mathrm{H} \mathrm{NMR}\left(\mathrm{D}_{2} \mathrm{O}\right): \delta 5.38\left(\mathrm{~d}, 1 \mathrm{H}, J_{1,2}=3.7 \mathrm{~Hz}\right.$, H-1'), $4.48\left(\mathrm{~d}, 1 \mathrm{H}, J_{1,2}=8.0 \mathrm{~Hz}, \mathrm{H}-1\right), 4.00 \sim 4.05\left(\mathrm{~m}, 1 \mathrm{H}, \mathrm{OCH}_{2} \mathrm{CH}_{2} \mathrm{OC}_{6} \mathrm{H}_{13}\right), 3.91$ $\left(\mathrm{dd}, 1 \mathrm{H}, J_{5,6 \mathrm{a}}=1.5 \mathrm{~Hz}, J_{6 \mathrm{a}, 6 \mathrm{~b}}=12.6 \mathrm{~Hz}, \mathrm{H}-6 \mathrm{a}\right), 3.75 \sim 3.85$ (m, 4H, H-3, H-6’a, H-6b, 
$\mathrm{OCH}_{2} \mathrm{CH}_{2} \mathrm{OC}_{6} \mathrm{H}_{13}$ ), 3.68 3.73 (m, 4H, H-5', H-6'b, $\mathrm{OCH}_{2} \mathrm{CH}_{2} \mathrm{OC}_{6} \mathrm{H}_{13}$ ), $3.59 \sim 3.67$ (m, 2H, H-3', H-4'), 3.53 3.58 (m, 4H, H-5, H-2', $\left.\mathrm{OCH}_{2}\left(\mathrm{CH}_{2}\right)_{4} \mathrm{CH}_{3}\right), 3.39$ (dd, $1 \mathrm{H}$, $J_{3,4}=J_{4,5}=9.4 \mathrm{~Hz}, \mathrm{H}-4$ '), $3.31\left(\mathrm{dd}, 1 \mathrm{H}, J_{2,3}=9.2 \mathrm{~Hz}, \mathrm{H}-2\right), 1.53 \sim 1.60(\mathrm{~m}, 2 \mathrm{H}$, $\left.\mathrm{OCH}_{2} \mathrm{CH}_{2}\left(\mathrm{CH}_{2}\right)_{3} \mathrm{CH}_{3}\right), 1.23 \sim 1.35\left(\mathrm{~m}, 6 \mathrm{H}, \mathrm{O}\left(\mathrm{CH}_{2}\right)_{2}\left(\mathrm{CH}_{2}\right)_{3} \mathrm{CH}_{3}\right), 0.85(\mathrm{t}, 3 \mathrm{H}, J=6.6$ $\left.\mathrm{Hz}, \mathrm{CH}_{3}\right)$. HRMS (ESI)m/z: calcd for $\mathrm{C}_{20} \mathrm{H}_{38} \mathrm{O}_{12} \mathrm{Na}^{+}(\mathrm{M}+\mathrm{Na})^{+}$493.22555, found 493.22528.

\subsection{Heptoxyethyl $\beta$ - $D$-maltoside}

Yield 82.5\%; $[\alpha]_{\mathrm{D}}^{20}+58.2^{\circ}\left(\mathrm{c} 1.0, \mathrm{CH}_{3} \mathrm{OH}\right) .{ }^{1} \mathrm{H}$ NMR $\left(\mathrm{D}_{2} \mathrm{O}\right): \delta 5.38\left(\mathrm{~d}, 1 \mathrm{H}, J_{1,2}=\right.$ $3.8 \mathrm{~Hz}, \mathrm{H}-1$ '), 4.48 (d, $\left.1 \mathrm{H}, J_{1,2}=8.0 \mathrm{~Hz}, \mathrm{H}-1\right), 4.00 \sim 4.05\left(\mathrm{~m}, 1 \mathrm{H}, \mathrm{OCH}_{2} \mathrm{CH}_{2} \mathrm{OC}_{7} \mathrm{H}_{15}\right)$, $3.91\left(\mathrm{dd}, 1 \mathrm{H}, J_{5,6 \mathrm{a}}=1.4 \mathrm{~Hz}, J_{6 \mathrm{a}, 6 \mathrm{~b}}=12.1 \mathrm{~Hz}, \mathrm{H}-6 \mathrm{a}\right), 3.75 \sim 3.85(\mathrm{~m}, 4 \mathrm{H}, \mathrm{H}-3, \mathrm{H}-6$ 'a, H-6b, $\left.\mathrm{OCH}_{2} \mathrm{CH}_{2} \mathrm{OC}_{7} \mathrm{H}_{15}\right), 3.67$ 3.74 (m, 4H, H-5', H-6'b, $\left.\mathrm{OCH}_{2} \mathrm{CH}_{2} \mathrm{OC}_{7} \mathrm{H}_{15}\right), 3.59$ $\sim 3.67$ (m, 2H, H-3', H-4), $3.53 \sim 3.58$ (m, 4H, H-5, H-2', $\left.\mathrm{OCH}_{2}\left(\mathrm{CH}_{2}\right)_{5} \mathrm{CH}_{3}\right), 3.39$ (dd, $\left.1 \mathrm{H}, J_{3,4}=J_{4,5}=9.4 \mathrm{~Hz}, \mathrm{H}-4^{\prime}\right), 3.31\left(\mathrm{dd}, 1 \mathrm{H}, J_{2,3}=9.2 \mathrm{~Hz}, \mathrm{H}-2\right), 1.54 \sim 1.60(\mathrm{~m}, 2 \mathrm{H}$, $\left.\mathrm{OCH}_{2} \mathrm{CH}_{2}\left(\mathrm{CH}_{2}\right)_{4} \mathrm{CH}_{3}\right), 1.23 \sim 1.35\left(\mathrm{~m}, 8 \mathrm{H}, \mathrm{O}\left(\mathrm{CH}_{2}\right)_{2}\left(\mathrm{CH}_{2}\right)_{4} \mathrm{CH}_{3}\right), 0.84(\mathrm{t}, 3 \mathrm{H}, J=6.8$ $\mathrm{Hz}, \mathrm{CH}_{3}$ ). HRMS (ESI)m/z: calcd for $\mathrm{C}_{21} \mathrm{H}_{40} \mathrm{O}_{12} \mathrm{Na}^{+}(\mathrm{M}+\mathrm{Na})^{+}$507.24120, found 507.24112 .

\subsection{Octoxyethyl $\beta$ - $D$-maltoside}

Yield 84.1\%; $[\alpha]_{\mathrm{D}}^{20}+60.9^{\circ}\left(\mathrm{c} 1.0, \mathrm{CH}_{3} \mathrm{OH}\right) .{ }^{1} \mathrm{H}$ NMR $\left(\mathrm{D}_{2} \mathrm{O}\right): \delta 5.34\left(\mathrm{~d}, 1 \mathrm{H}, J_{1,2}=\right.$ $3.6 \mathrm{~Hz}, \mathrm{H}-1$ '), $4.43\left(\mathrm{~d}, 1 \mathrm{H}, J_{1,2}=8.0 \mathrm{~Hz}, \mathrm{H}-1\right), 3.97 \sim 4.02\left(\mathrm{~m}, 1 \mathrm{H}, \mathrm{OCH}_{2} \mathrm{CH}_{2} \mathrm{OC}_{8} \mathrm{H}_{17}\right)$, $3.88\left(\mathrm{dd}, 1 \mathrm{H}, J_{5,6 \mathrm{a}}=1.1 \mathrm{~Hz}, J_{6 \mathrm{a}, 6 \mathrm{~b}}=11.9 \mathrm{~Hz}, \mathrm{H}-6 \mathrm{a}\right), 3.72 \sim 3.82(\mathrm{~m}, 4 \mathrm{H}, \mathrm{H}-3, \mathrm{H}-6$ ' a, H-6b, $\left.\mathrm{OCH}_{2} \mathrm{CH}_{2} \mathrm{OC}_{8} \mathrm{H}_{17}\right), 3.63 \sim 3.71$ (m, 4H, H-5', H-6'b, $\left.\mathrm{OCH}_{2} \mathrm{CH}_{2} \mathrm{OC}_{8} \mathrm{H}_{17}\right), 3.57$ 3.63 (m, 2H, H-3', H-4), 3.48 3.55 (m, 4H, H-5, H-2', $\left.\mathrm{CH}_{2}\left(\mathrm{CH}_{2}\right)_{6} \mathrm{CH}_{3}\right), 3.37$ (dd, $1 \mathrm{H}, J_{3,4}=J_{4,5}=9.4 \mathrm{~Hz}, \mathrm{H}-4$ ') $3.29\left(\mathrm{dd}, 1 \mathrm{H}, J_{2,3}=9.2 \mathrm{~Hz}, \mathrm{H}-2\right), 1.51 \sim 1.58(\mathrm{~m}, 2 \mathrm{H}$, $\left.\mathrm{OCH}_{2} \mathrm{CH}_{2}\left(\mathrm{CH}_{2}\right)_{5} \mathrm{CH}_{3}\right), 1.21 \sim 1.32\left(\mathrm{~m}, 10 \mathrm{H}, \mathrm{O}\left(\mathrm{CH}_{2}\right)_{2}\left(\mathrm{CH}_{2}\right)_{5} \mathrm{CH}_{3}\right), 0.82(\mathrm{t}, 3 \mathrm{H}, J=6.5$ $\mathrm{Hz}, \mathrm{CH}_{3}$ ). HRMS (ESI)m/z: calcd for $\mathrm{C}_{22} \mathrm{H}_{42} \mathrm{O}_{12} \mathrm{Na}^{+}(\mathrm{M}+\mathrm{Na})^{+} 521.25685$, found 521.25702 .

\subsection{Nonoxyethyl $\beta$ - $D$-maltoside}

Yield 83.8\%; $[\alpha]_{\mathrm{D}}^{20}+56.5^{\circ}\left(\mathrm{c} 1.0, \mathrm{CH}_{3} \mathrm{OH}\right) .{ }^{1} \mathrm{H}$ NMR $\left(\mathrm{D}_{2} \mathrm{O}\right): \delta 5.38\left(\mathrm{~d}, 1 \mathrm{H}, J_{1,2}=\right.$ $3.6 \mathrm{~Hz}, \mathrm{H}-1$ '), 4.47 (d, $\left.1 \mathrm{H}, J_{1,2}=7.8 \mathrm{~Hz}, \mathrm{H}-1\right), 4.01 \sim 4.08\left(\mathrm{~m}, 1 \mathrm{H}, \mathrm{OCH}_{2} \mathrm{CH}_{2} \mathrm{OC}_{9} \mathrm{H}_{19}\right.$ ), $3.92\left(\mathrm{dd}, 1 \mathrm{H}, J_{5,6 \mathrm{a}}=0.5 \mathrm{~Hz}, J_{6 \mathrm{a}, 6 \mathrm{~b}}=11.6 \mathrm{~Hz}, \mathrm{H}-6 \mathrm{a}\right), 3.78 \sim 3.87(\mathrm{~m}, 4 \mathrm{H}, \mathrm{H}-3, \mathrm{H}-6$ 'a, 
H-6b, $\left.\mathrm{OCH}_{2} \mathrm{CH}_{2} \mathrm{OC}_{9} \mathrm{H}_{19}\right), 3.68 \sim 3.77$ ( $\mathrm{m}, 4 \mathrm{H}, \mathrm{H}-5$ ', H-6'b, $\left.\mathrm{OCH}_{2} \mathrm{CH}_{2} \mathrm{OC}_{9} \mathrm{H}_{19}\right), 3.62$ 3.67 (m, 2H, H-3', H-4), 3.52 3.61 (m, 4H, H-5, H-2', $\left.\mathrm{OCH}_{2}\left(\mathrm{CH}_{2}\right)_{7} \mathrm{CH}_{3}\right), 3.43$ (dd, $\left.1 \mathrm{H}, J_{3,4}=J_{4,5}=9.3 \mathrm{~Hz}, \mathrm{H}-4^{\prime}\right), 3.36\left(\mathrm{dd}, 1 \mathrm{H}, J_{2,3}=8.8 \mathrm{~Hz}, \mathrm{H}-2\right), 1.56 \sim 1.64(\mathrm{~m}, 2 \mathrm{H}$, $\left.\mathrm{OCH}_{2} \mathrm{CH}_{2}\left(\mathrm{CH}_{2}\right)_{6} \mathrm{CH}_{3}\right), 1.23 \sim 1.36\left(\mathrm{~m}, 12 \mathrm{H}, \mathrm{O}\left(\mathrm{CH}_{2}\right)_{2}\left(\mathrm{CH}_{2}\right)_{6} \mathrm{CH}_{3}\right), 0.88(\mathrm{t}, 3 \mathrm{H}, J=6.0$ $\mathrm{Hz}, \mathrm{CH}_{3}$ ). HRMS (ESI)m/z: calcd for $\mathrm{C}_{23} \mathrm{H}_{44} \mathrm{O}_{12} \mathrm{Na}^{+}(\mathrm{M}+\mathrm{Na})^{+}$535.27250, found 535.27295 .

\subsection{Decoxyethyl $\beta$ - $D$-maltoside}

Yield 80.5\%; $[\alpha]_{\mathrm{D}}^{20}+69.9^{\circ}\left(\mathrm{c} 1.0, \mathrm{CH}_{3} \mathrm{OH}\right) .{ }^{1} \mathrm{H}$ NMR (DMSO-d6/D $\left.\mathrm{D}_{2} \mathrm{O}\right): \delta 5.00(\mathrm{~d}$, $\left.1 \mathrm{H}, J_{1,2}=3.8 \mathrm{~Hz}, \mathrm{H}-1^{\prime}\right), 4.19\left(\mathrm{~d}, 1 \mathrm{H}, J_{1,2}=7.8 \mathrm{~Hz}, \mathrm{H}-1\right), 3.79 \sim 3.85(\mathrm{~m}, 1 \mathrm{H}$, $\left.\mathrm{OCH}_{2} \mathrm{CH}_{2} \mathrm{OC}_{10} \mathrm{H}_{21}\right), 3.68\left(\mathrm{dd}, 1 \mathrm{H}, J_{5,6 \mathrm{~b}}=1.8 \mathrm{~Hz}, J_{6 \mathrm{a}, 6 \mathrm{~b}}=12.3 \mathrm{~Hz}, \mathrm{H}-6 \mathrm{a}\right), 3.51 \sim 3.61$ $\left(\mathrm{m}, \quad 3 \mathrm{H}, \quad \mathrm{H}-6\right.$ 'a, $\left.\mathrm{OCH}_{2} \mathrm{CH}_{2} \mathrm{OC}_{10} \mathrm{H}_{21}, \mathrm{H}-6 \mathrm{~b}\right), 3.44 \sim 3.51$ (m, 4H, H-6’b, $\left.\mathrm{OCH}_{2} \mathrm{CH}_{2} \mathrm{OC}_{10} \mathrm{H}_{21}, \mathrm{H}_{-5}{ }^{\prime}\right), 3.38 \sim 3.43(\mathrm{~m}, 2 \mathrm{H}, \mathrm{H}-3, \mathrm{H}-4), 3.26 \sim 3.36(\mathrm{~m}, 3 \mathrm{H}$, $\mathrm{OCH}_{2} \mathrm{CH}_{2} \mathrm{OCH}_{2}\left(\mathrm{CH}_{2}\right)_{8} \mathrm{CH}_{3}, \mathrm{H}-3^{\prime}$ '), $3.21 \sim 3.25$ (m, 2H, H-5, H-2'), 3.05 (dd, 1H, J3,4 $\left.=J_{4,5}=9.0 \mathrm{~Hz}, \mathrm{H}-4^{\prime}\right), 3.00\left(\mathrm{dd}, 1 \mathrm{H}, J_{2,3}=8.9 \mathrm{~Hz}, \mathrm{H}-2\right), 1.41 \sim 1.48(\mathrm{~m}, 2 \mathrm{H}$, $\left.\mathrm{OCH}_{2} \mathrm{CH}_{2}\left(\mathrm{CH}_{2}\right)_{7} \mathrm{CH}_{3}\right), 1.17 \sim 1.27\left(\mathrm{~m}, 14 \mathrm{H}, \mathrm{O}\left(\mathrm{CH}_{2}\right)_{2}\left(\mathrm{CH}_{2}\right)_{7} \mathrm{CH}_{3}\right), 0.82(\mathrm{t}, 3 \mathrm{H}, J=6.8$ $\mathrm{Hz}, \mathrm{CH}_{3}$ ). HRMS (ESI)m/z: calcd for $\mathrm{C}_{24} \mathrm{H}_{46} \mathrm{O}_{12} \mathrm{Na}^{+}(\mathrm{M}+\mathrm{Na})^{+} 549.28815$, found 549.28870 .

\subsection{Dodecoxyethyl $\beta$ - $D$-maltoside}

Yield 82.6\%; $[\alpha]_{\mathrm{D}}^{20}+55.6^{\circ}\left(\mathrm{c} 1.0, \mathrm{CH}_{3} \mathrm{OH}\right) .{ }^{1} \mathrm{H}$ NMR (DMSO-d6/D $\left.2 \mathrm{O}\right): \delta 5.00(\mathrm{~d}$, $\left.1 \mathrm{H}, J_{1,2}=3.6 \mathrm{~Hz}, \mathrm{H}-1^{\prime}\right), 4.18\left(\mathrm{~d}, 1 \mathrm{H}, J_{1,2}=7.8 \mathrm{~Hz}, \mathrm{H}-1\right), 3.80 \sim 3.85(\mathrm{~m}, 1 \mathrm{H}$, $\left.\mathrm{OCH}_{2} \mathrm{CH}_{2} \mathrm{OC}_{12} \mathrm{H}_{25}\right), 3.68\left(\mathrm{dd}, 1 \mathrm{H}, J_{5,6 \mathrm{a}}=1.7 \mathrm{~Hz}, J_{6 \mathrm{a}, 6 \mathrm{~b}}=12.3 \mathrm{~Hz}, \mathrm{H}-6 \mathrm{a}\right), 3.51 \sim 3.61$ $\left(\mathrm{m}, \quad 3 \mathrm{H}, \quad \mathrm{H}-6\right.$ 'a, $\left.\mathrm{OCH}_{2} \mathrm{CH}_{2} \mathrm{OC}_{12} \mathrm{H}_{25}, \mathrm{H}-6 \mathrm{~b}\right), 3.43 \sim 3.51(\mathrm{~m}, 4 \mathrm{H}, \quad \mathrm{H}-6$ 'b, $\mathrm{OCH}_{2} \mathrm{CH}_{2} \mathrm{OC}_{12} \mathrm{H}_{25}, \mathrm{H}-5$ '), $3.36 \sim 3.41(\mathrm{~m}, 2 \mathrm{H}, \mathrm{H}-3, \mathrm{H}-4), 3.26 \sim 3.34(\mathrm{~m}, 3 \mathrm{H}$, $\mathrm{OCH}_{2} \mathrm{CH}_{2} \mathrm{OCH}_{2}\left(\mathrm{CH}_{2}\right)_{10} \mathrm{CH}_{3}, \mathrm{H}-3$ '), $3.20 \sim 3.25$ (m, 2H, H-5, H-2'), 3.05 (dd, 1H, J3,4 $\left.=J_{4,5}=9.0 \mathrm{~Hz}, \mathrm{H}-4^{\prime}\right), 3.01\left(\mathrm{dd}, 1 \mathrm{H}, J_{2,3}=8.9 \mathrm{~Hz}, \mathrm{H}-2\right), 1.39 \sim 1.46(\mathrm{~m}, 2 \mathrm{H}$, $\left.\mathrm{OCH}_{2} \mathrm{CH}_{2}\left(\mathrm{CH}_{2}\right)_{9} \mathrm{CH}_{3}\right), 1.15 \sim 1.25\left(\mathrm{~m}, 18 \mathrm{H}, \mathrm{O}\left(\mathrm{CH}_{2}\right)_{2}\left(\mathrm{CH}_{2}\right)_{9} \mathrm{CH}_{3}\right), 0.80(\mathrm{t}, 3 \mathrm{H}, J=6.6$ $\mathrm{Hz}, \mathrm{CH}_{3}$ ). HRMS (ESI)m/z: calcd for $\mathrm{C}_{26} \mathrm{H}_{50} \mathrm{O}_{12} \mathrm{Na}^{+}(\mathrm{M}+\mathrm{Na})^{+}$577.31945, found 577.31982 .

\subsection{Tetradecoxyethyl $\beta$ - $D$-maltoside}

Yield 81.2\%; $[\alpha]_{\mathrm{D}}^{20}+67.4^{\circ}\left(\mathrm{c} 1.0, \mathrm{CH}_{3} \mathrm{OH}\right) .{ }^{1} \mathrm{H}$ NMR $\left(\mathrm{DMSO}-\mathrm{d} 6 / \mathrm{D}_{2} \mathrm{O}\right): \delta 5.01(\mathrm{~d}$, 
$\left.1 \mathrm{H}, J_{1,2}=3.8 \mathrm{~Hz}, \mathrm{H}-1^{\prime}\right), 4.18\left(\mathrm{~d}, 1 \mathrm{H}, J_{1,2}=7.8 \mathrm{~Hz}, \mathrm{H}-1\right), 3.79 \sim 3.84(\mathrm{~m}, 1 \mathrm{H}$, $\left.\mathrm{OCH}_{2} \mathrm{CH}_{2} \mathrm{OC}_{14} \mathrm{H}_{29}\right), 3.68\left(\mathrm{dd}, 1 \mathrm{H}, J_{5,6 \mathrm{a}}=1.7 \mathrm{~Hz}, J_{6 \mathrm{a}, 6 \mathrm{~b}}=12.2 \mathrm{~Hz}, \mathrm{H}-6 \mathrm{a}\right), 3.52 \sim 3.61$ $\left(\mathrm{m}, \quad 3 \mathrm{H}, \quad \mathrm{H}-6\right.$ 'a, $\left.\mathrm{OCH}_{2} \mathrm{CH}_{2} \mathrm{OC}_{14} \mathrm{H}_{29}, \quad \mathrm{H}-6 \mathrm{~b}\right), 3.43 \sim 3.50$ (m, 4H, H-6’b, $\left.\mathrm{OCH}_{2} \mathrm{CH}_{2} \mathrm{OC}_{14} \mathrm{H}_{29}, \mathrm{H}_{-5}{ }^{\prime}\right), 3.36 \sim 3.41(\mathrm{~m}, 2 \mathrm{H}, \mathrm{H}-3, \mathrm{H}-4), 3.26 \sim 3.34(\mathrm{~m}, 3 \mathrm{H}$, $\mathrm{OCH}_{2} \mathrm{CH}_{2} \mathrm{OCH}_{2}\left(\mathrm{CH}_{2}\right)_{12} \mathrm{CH}_{3}, \mathrm{H}-3$ '), $3.20 \sim 3.25$ (m, 2H, H-5, H-2'), 3.06 (dd, 1H, J3,4 $=J_{4,5}=9.2 \mathrm{~Hz}, \mathrm{H}-4$ ') $3.01\left(\mathrm{dd}, 1 \mathrm{H}, J_{2,3}=9.1 \mathrm{~Hz}, \mathrm{H}-2\right), 1.39 \sim 1.46(\mathrm{~m}, 2 \mathrm{H}$, $\left.\mathrm{OCH}_{2} \mathrm{CH}_{2}\left(\mathrm{CH}_{2}\right)_{11} \mathrm{CH}_{3}\right), 1.15 \sim 1.25\left(\mathrm{~m}, 22 \mathrm{H}, \mathrm{O}\left(\mathrm{CH}_{2}\right)_{2}\left(\mathrm{CH}_{2}\right)_{11} \mathrm{CH}_{3}\right), 0.80(\mathrm{t}, 3 \mathrm{H}, J=$ $6.8 \mathrm{~Hz}, \mathrm{CH}_{3}$ ). HRMS (ESI)m/z: calcd for $\mathrm{C}_{28} \mathrm{H}_{54} \mathrm{O}_{12} \mathrm{Na}^{+}(\mathrm{M}+\mathrm{Na})^{+} 605.35075$, found 605.35046 .

\section{8 hexadecoxyethyl $\beta$ - $D$-maltoside}

Yield 80.6\%; $[\alpha]_{\mathrm{D}}^{20}+43.8^{\circ}\left(\mathrm{c} 1.0, \mathrm{CH}_{3} \mathrm{OH}\right) .{ }^{1} \mathrm{H}$ NMR (DMSO-d6/ $\left.\mathrm{D}_{2} \mathrm{O}\right): \delta 5.00(\mathrm{~d}$, $\left.1 \mathrm{H}, J_{1,2}=3.9 \mathrm{~Hz}, \mathrm{H}-1^{\prime}\right), 4.18\left(\mathrm{~d}, 1 \mathrm{H}, J_{1,2}=7.8 \mathrm{~Hz}, \mathrm{H}-1\right), 3.79 \sim 3.84(\mathrm{~m}, 1 \mathrm{H}$, $\left.\mathrm{OCH}_{2} \mathrm{CH}_{2} \mathrm{OC}_{16} \mathrm{H}_{33}\right), 3.67\left(\mathrm{dd}, 1 \mathrm{H}, J_{5,6 \mathrm{a}}=1.4 \mathrm{~Hz}, J_{6 \mathrm{a}, 6 \mathrm{~b}}=12.0 \mathrm{~Hz}, \mathrm{H}-6 \mathrm{a}\right), 3.52 \sim 3.61$ $\left(\mathrm{m}, \quad 3 \mathrm{H}, \quad \mathrm{H}-6\right.$ 'a, $\left.\mathrm{OCH}_{2} \mathrm{CH}_{2} \mathrm{OC}_{16} \mathrm{H}_{33}, \mathrm{H}-6 \mathrm{~b}\right), 3.43 \sim 3.50$ (m, 4H, H-6’b, $\left.\mathrm{OCH}_{2} \mathrm{CH}_{2} \mathrm{OC}_{16} \mathrm{H}_{33}, \mathrm{H}_{-} 5^{\prime}\right), 3.39 \sim 3.41(\mathrm{~m}, 2 \mathrm{H}, \mathrm{H}-3, \mathrm{H}-4), 3.26 \sim 3.34(\mathrm{~m}, 3 \mathrm{H}$, $\mathrm{OCH}_{2} \mathrm{CH}_{2} \mathrm{OCH}_{2}\left(\mathrm{CH}_{2}\right)_{14} \mathrm{CH}_{3}, \mathrm{H}-3$ '), $3.20 \sim 3.25$ (m, 2H, H-5, H-2'), 3.06 (dd, 1H, J3,4 $\left.=J_{4,5}=9.1 \mathrm{~Hz}, \mathrm{H}-4^{\prime}\right), 3.01\left(\mathrm{dd}, 1 \mathrm{H}, J_{2,3}=8.9 \mathrm{~Hz}, \mathrm{H}-2\right), 1.39 \sim 1.46(\mathrm{~m}, 2 \mathrm{H}$, $\left.\mathrm{OCH}_{2} \mathrm{CH}_{2}\left(\mathrm{CH}_{2}\right)_{13} \mathrm{CH}_{3}\right), 1.13 \sim 1.25\left(\mathrm{~m}, 26 \mathrm{H}, \mathrm{O}\left(\mathrm{CH}_{2}\right)_{2}\left(\mathrm{CH}_{2}\right)_{13} \mathrm{CH}_{3}\right), 0.80(\mathrm{t}, 3 \mathrm{H}, J=$ $\left.6.8 \mathrm{~Hz}, \mathrm{CH}_{3}\right)$. HRMS (ESI)m/z: calcd for $\mathrm{C}_{30} \mathrm{H}_{58} \mathrm{O}_{12} \mathrm{Na}^{+}(\mathrm{M}+\mathrm{Na})^{+} 633.38205$, found 633.38245 . 